\title{
The relationship between tissue oxygenation and redox status using magnetic resonance imaging
}

\author{
FUMINORI HYODO, RYAN M. DAVIS, EMI HYODO, SHINGO MATSUMOTO, \\ MURALI C. KRISHNA and JAMES B. MITCHELL
}

Radiation Biology Branch, Center for Cancer Research, National Cancer Institute, NIH, Bethesda, MD, USA

Received July 26, 2012; Accepted September 12, 2012

DOI: 10.3892/ijo.2012.1638

\begin{abstract}
The recent development of a bi-modality magnetic resonance imaging/electron paramagnetic resonance imaging (MRI/EPRI) platform has enabled longitudinal monitoring of both tumor oxygenation and redox status in murine cancer models. The current study used this imaging platform to test the hypothesis that a more reducing tumor microenvironment accompanies the development of tumor hypoxia. To test this, the redox status of the tumor was measured using Tempol as a redox-sensitive MRI contrast agent, and tumor hypoxia was measured with Oxo63, which is an oxygen-sensitive EPRI spin probe. Images were acquired every 1-2 days in mice bearing SCCVII tumors. The median $\mathrm{pO}_{2}$ decreased from $14 \mathrm{mmHg}$ at 7 days after tumor implantation to $7 \mathrm{mmHg}$ at 15 days after implantation. Additionally, the hypoxic fraction, defined as the percentage of the tumor that exhibited a $\mathrm{pO}_{2}<10 \mathrm{mmHg}$, increased with tumor size (from $10 \%$ at $500 \mathrm{~mm}^{3}$ to $60 \%$ at $3,500 \mathrm{~mm}^{3}$ ). The rate of Tempol reduction increased as a function of tumor volume $\left(0.4 \mathrm{~min}^{-1}\right.$ at $500 \mathrm{~mm}^{3}$ to $1.7 \mathrm{~min}^{-1}$ at $\left.3,500 \mathrm{~mm}^{3}\right)$, suggesting that the tumor microenvironment became more reduced as the tumor grew. The results show that rapid Tempol reduction correlates with decreased tumor oxygenation, and that the Tempol decay rate constant may be a surrogate marker for tumor hypoxia.
\end{abstract}

\section{Introduction}

Tumor hypoxia is prognostic for poor response to cancer therapy. The prognostic value of tumor hypoxia has been demonstrated in radiotherapy studies of human head and neck $(1,2)$ and uterine cervix (3) tumors, which show that patients with less hypoxic tumors had a better chance of overall or disease-free survival than did patients with more hypoxic tumors. Furthermore,

Correspondence to: Dr James B. Mitchell, Radiation Biology Branch, NCI, Bldg. 10, Room B3-B69, 9000 Rockville Pike, Bethesda, MD 20892, USA

E-mail: jbm@helix.nih.gov

Key words: redox status, hypoxia, electron paramagnetic resonance imaging, magnetic resonance imaging, nitroxide, non-invasive imaging, Tempol some chemotherapeutic agents such as bleomycin and doxorubicin exhibit a cytotoxicity that is strongly oxygen dependent, suggesting that hypoxia may be a prognostic factor for chemotherapeutic response as well (4-6). Therefore, development of methods allowing measurement of hypoxia in human patients may allow physicians to better manage tumors that exhibit considerable hypoxia.

Ilangovan et al demonstrated in tumor-bearing mice that carbogen breathing increased the oxygenation in the tumor, and that this increased oxygenation was related to a decreased rate of nitroxide reduction (7). Magnetic resonance imaging (MRI) can accurately measure nitroxide reduction rates $(8,9)$, suggesting that nitroxide contrast agents could serve as an MRI-based assessment of tumor oxygenation. Although the study by Ilangovan showed that nitroxides were sensitive to oxygenation changes during carbogen breathing, another use of nitroxides would be to detect hypoxia in tumors (7). In this case, it is expected that hypoxia will have the opposite effect of carbogen on the rate of nitroxide reduction. Namely, it is expected that greater hypoxia will be associated with a greater rate of nitroxide reduction.

The purpose of this study was to test if there is a relationship between the reduction rate of Tempol as measured with MRI and the hypoxic fraction of a tumor. The hypoxic fraction of the tumor was measured using electron paramagnetic resonance (EPR) imaging and the triarylmethyl (TAM) spin probe Oxo63 and the reduction rate of Tempol was measured with a $7 \mathrm{~T}$ small animal MRI scanner.

\section{Materials and methods}

Chemicals. The triarylmethyl (TAM) radical Oxo63 was obtained from GE healthcare. Tempol (4-hydroxy-2,2,6,6,-tetramethyl-1-piperidynyloxyl) was purchased from Sigma-Aldrich (St. Louis, MO, USA).

Animals. C3HHenCrMTV- mice were obtained from the Frederick Cancer Research Center, Animal Production (Frederick, MD, USA). Mice were housed in a climate controlled circadian rhythm adjusted room and were allowed access to food and water ad libitum. The body weight of the mice at the time of imaging was 22-30 g. SCCVII (murine squamous cell carcinoma) cells $\left(2-3 \times 10^{5}\right)$ were injected 7-15 days before imaging. Experiments were carried out in compliance with the Guide for 
the Care and Use of Laboratory Animal Resources (National Research Council, 1996) and approved by the National Cancer Institute Animal Care and Use Committee.

Animal experiment protocol. Starting 7 days after injection of SCCVII cells, mice were imaged every 1-2 days. For anesthesia during imaging, a mixture of isofluorane (4\% to induce, $1-2 \%$ to maintain) and medical air $(750 \mathrm{ml} / \mathrm{min})$ was blown into a nose-cone fitted to the animal's head. For EPR measurements, the mouse was placed on a platform with its legs hanging downward into a vertical coil. For MRI measurements, the mouse was placed on a cradle, which was then placed inside the horizontal bore of the magnet. For both EPR and MRI experiments, a pressure transducer (SA Instruments Inc.) was used to monitor the breathing rate, and a rectal thermocouple was used to monitor the core body temperature. Warm air was blown over the mouse to maintain its temperature. The breathing rate was kept at $60 \pm 10$ breaths per min and the body temperature was maintained at $37 \pm 1^{\circ} \mathrm{C}$. To enable injection of contrast agents during imaging, a catheter was made by inserting a 30.5-gauge needle tip into polyethylene (PE-10) tubing. Using this catheter, the tail vein of the mouse was cannulated. Oxo63 (10 $\mu \mathrm{l} / \mathrm{g}$ body weight of $75 \mathrm{mM}$ solution) or Tempol ( $5 \mu \mathrm{l} / \mathrm{g}$ body weight of $150 \mathrm{mM}$ solution) was manually injected through the catheter.

Magnetic resonance imaging. Magnetic Resonance Imaging was performed using a 4.7 Tesla small animal scanner (Bruker Bio-Spin MRI GmbH). After a survey scan, T2-weighted images were obtained using multi-slice multi-echo (MSME) sequence with a 10 -echo train and an echo time of $15 \mathrm{~min}$. SPGR (also referred as gradient echo fast imaging, GEFI) $(\mathrm{TR}=75 \mathrm{~ms}$, $\mathrm{TE}=3 \mathrm{~ms}, \mathrm{FA}=45^{\circ}, \mathrm{NEX}=2$ ) was employed to observe $\mathrm{T} 1$ effect. The scan time for 6 slices with the SPGR sequence was $20 \mathrm{sec}$. Other common image parameters are as follows; image resolution was $256 \times 256$, FOV was $3.2 \times 3.2 \mathrm{~cm}$, slice thickness was $2.0 \mathrm{~mm}$. Number of slices was 6 .

EPR oximetry. A pulsed (time domain) EPR imaging scanner was used to measure tissue $\mathrm{pO}_{2}$. Details of this method are described elsewhere (10). Briefly, Oxo63 is injected into the mouse via tail vein catheter. Imaging was initiated once the EPR signal intensity reached a steady state value, indicating that the Oxo63 radical had reached a steady state concentration in the tissue. $\mathrm{pO}_{2}$ mapping relies on the linear relationship between the $\mathrm{pO}_{2}$ of the tissue and linewidth of the TAM radical (Oxo63.) To quantify the linewidth in a voxel, several images with increasing readout delay are obtained. As the readout delay increases, the signal decreases in a manner that depends on the linewidth of the voxel. From the images with successively increasing readout delay, the linewidth of oxo63 is mapped over the tissue region. Finally, using the linewidth map, the $\mathrm{pO}_{2}$ is calculated from a calibration curve calculated in vitro. EPR imaging was performed in a $300 \mathrm{MHz}$ single point-imaging scanner. The imaging parameters were as follows: excitation pulse, $80 \mathrm{~ns}$, $80 \mathrm{~W}$; TR, $5.5 \mu \mathrm{s}$; flip angle, $70^{\circ} \mathrm{C}$; field gradient, 0.8 Gauss $/ \mathrm{cm}$, 1.0 Gauss $/ \mathrm{cm}, 1.2$ Gauss $/ \mathrm{cm}$; no. of gradient steps, $21 \times 21$, number of averages, 100,000 .

Statistical analyses. The statistical differences were estimated with TTEST function in the Microsoft Excel XP. The suitable
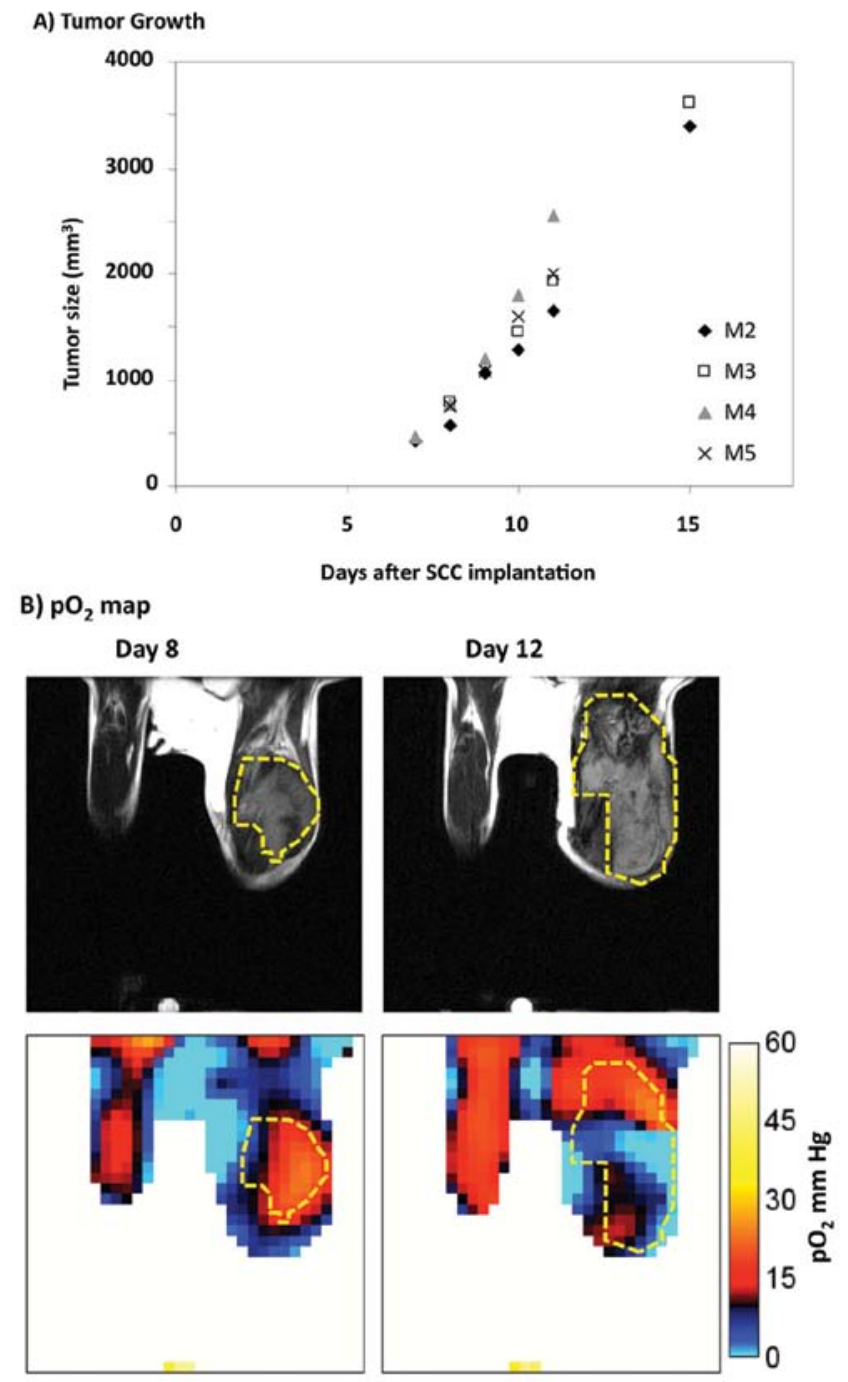

Figure 1. (A) SCCVII tumor size as a function of time after implantation. (B) T2-weighted anatomic image of a representative SCCVII tumor-bearing mouse at day 8 and day 12 (upper panels) and corresponding $\mathrm{pO}_{2}$ maps (bottom panels) acquired by EPRI. The tumor location is delineated by dashed yellow line on each of the images.

'type' for the test was selected according to the correspondence and variance of the data. Significances were estimated when p-value was less than 0.05 .

\section{Results}

The growth of the SCCVII tumor as a function of time after implantation (Fig. 1A). Between days 7 and 15, the volume of the tumor increased linearly with time from 500 to $3,500 \mathrm{~mm}^{3}$. Fig. 1B shows representative EPR-based $\mathrm{pO}_{2}$ maps (bottom panels) obtained at 8 and 12 days after tumor implantation. Comparison of the MRI T2-weighted images (top panels) at day 8 and day 12 clearly shows increase in tumor size with time. The $\mathrm{pO}_{2}$ maps overlaid with the MRI scans show significant hypoxia regions (black and blue areas on the images) within the tumor by day 12 . The $\mathrm{pO}_{2}$ maps as shown in Fig. 1B were converted into histograms and representative histograms for a representative mouse on days 7 and 15 are displayed in Fig. 2. These histograms show that over the 

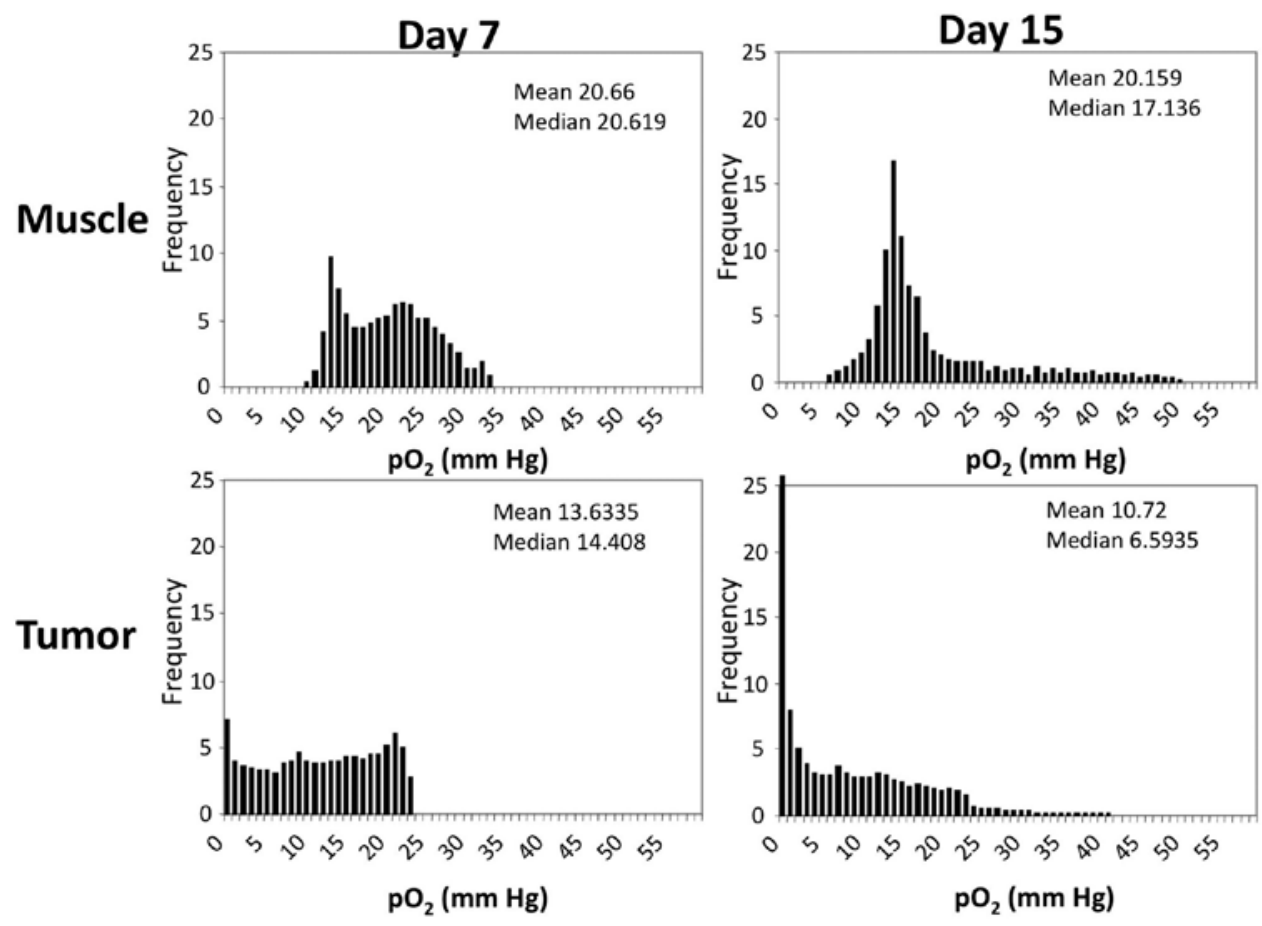

Figure 2. Comparison of muscle and tumor $\mathrm{pO}_{2}$ histograms at day 7 and day 15 from a representative SCCVII tumor-bearing mouse. Hypoxia increased substantially from day 7 to day 15 in the tumor.

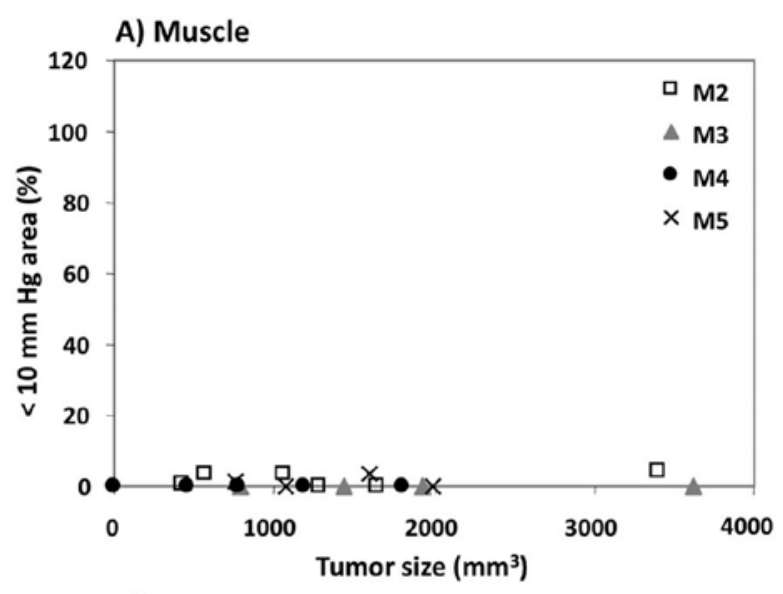

B) Tumor

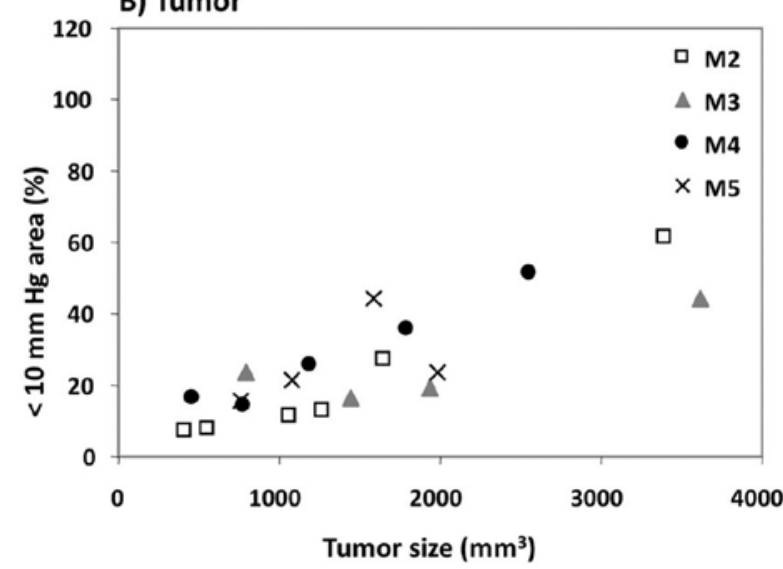

Figure 3. Hypoxic fraction (\% of tissue $<10 \mathrm{mmHg}$ oxygen) for muscle and SCCVII tumors as a function of tumor size. Normal muscle exhibited little to no hypoxia; whereas, as the SCCVII tumors grew, there was a substantial development of hypoxia. Data presented are for 4 mice followed serially with time. course of the study, a slight $3 \mathrm{mmHg}$ decrease in median $\mathrm{pO}_{2}$ was observed in the muscle, while a marked $8 \mathrm{mmHg}$ decrease in median $\mathrm{pO}_{2}$ was observed in the tumor. To test if the hypoxic fraction of the tumor changed as the tumor grew, the percentage of tumor volume with $\mathrm{pO}_{2}$ less than $10 \mathrm{mmHg}$ (i.e., the hypoxic fraction) was plotted as a function of tumor size (Fig. 3). Between days 7 and 15 of the study, the hypoxic fraction of the muscle remained well below 5\%. In contrast, the hypoxic fraction of the tumor increased linearly as the tumor progressed, reaching approximately $50 \%$ by the end of the study. Taken together with the data from Figs. 1 and 2 , these results strongly suggest that growth of the SCCVII tumor correlates with the development of hypoxic regions within the tumor.

The redox status of the tissue was monitored using Tempol as a redox-sensitive magnetic resonance imaging contrast agent. Fig. 4 shows a representative redox data set for a mouse taken at days 7 and 14 after tumor implantation. Fig. 4A shows T2-weighted images of the tumor region, with regions of interest (ROI) outlined with yellow lines for muscle and tumor. ROIs were used to calculate the rate of Tempol reduction. Fig. 4B shows the observed signal enhancement $40 \mathrm{sec}$ after Tempol injection. On both days 7 and 14, the rate of Tempol reduction was greater in tumor tissue than in muscle. As the tumor grew in size, the rate at which muscle reduced Tempol did not appreciably change, while the rate at which the tumor reduced Tempol more than doubled. Fig. 5A summarizes the changes in tumor volume and Tempol reduction rates for 4 mice. As the tumor volume increased, the rate at which the tumor reduced Tempol also increased. As for muscle, Tempol reduction essentially the same over the 14 days. Finally, the rate of Tempol reduction and 


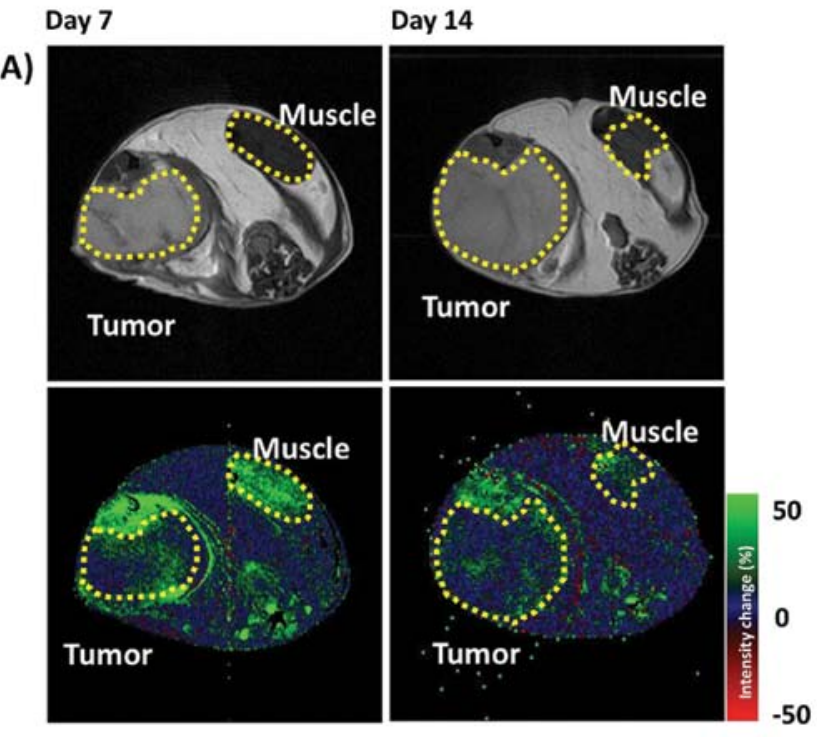

B)
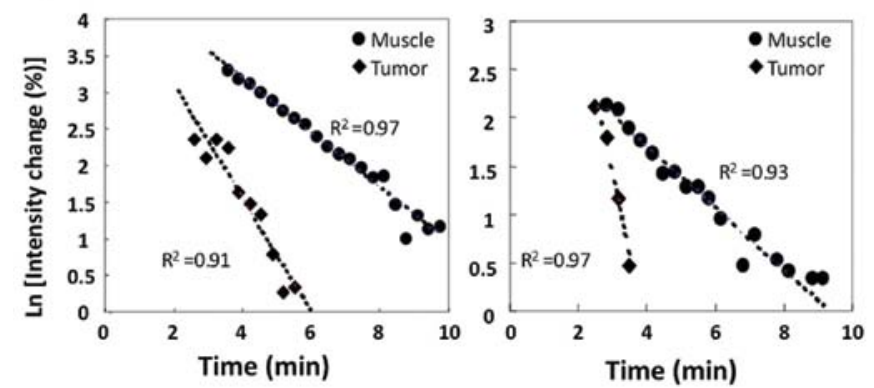

Figure 4. (A) T2-weighted anatomic images of a representative SCCVII tumorbearing mouse at day 7 and day 14 (upper panels) and corresponding Tempol redox maps (bottom panels) acquired by MRI. Tumor and muscle locations are delineated by dashed yellow line on each of the images. (B) The rate of Tempol decay as a function of time after injection for muscle and tumor ROIs. Linear regression lines are shown on the plots with the respective $\mathrm{R} 2$ values indicated.

the hypoxic fraction of a SCCVII tumor were plotted as a function of tumor size as shown on Fig. 5B. This graph shows that larger tumors have hypoxic fractions ranging from $40 \%-60 \%$ and reduction rates varying from 1.2-1.6 $\mathrm{min}^{-1}$, while smaller tumors have hypoxic fractions of only $5-20 \%$ and reduction rates of only $0.4-0.6 \mathrm{~min}^{-1}$. This comparison suggests that the rate of Tempol reduction and the hypoxic fraction of a tumor were positively correlated.

\section{Discussion}

The purpose of this study was to determine if the hypoxic fraction of a SCCVII tumor correlated with the rate of Tempol reduction in the tumor. It was found that both the rate of Tempol reduction as well as the hypoxic fraction (the fraction of voxels with less than $10 \mathrm{mmHg}$ ) increased as the tumor grew (Fig. 5B). This implies a correlation between the rate of Tempol reduction and the hypoxic fraction. Because high tumor hypoxia predicts a decreased likelihood of survival during clinical cancer therapy (1-3), the data from the current study suggest that rapid Tempol reduction may be indicative of a poor prognosis for cancer therapy.

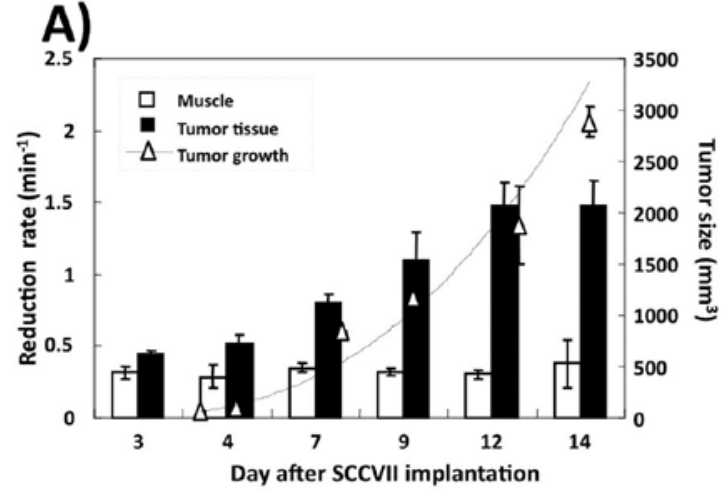

B)

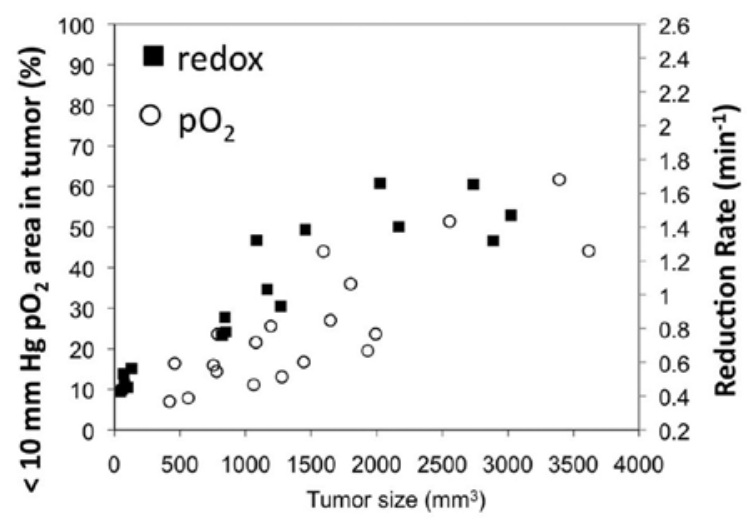

Figure 5. (A) Relationship between Tempol reduction rate and tumor size for muscle and tumor tissue as a function of time, $n=4$. (B) Relationship between hypoxia (\% of tumor $<10 \mathrm{mmHg}$ ) and Tempol reduction rates as a function of tumor size.

In this study, it was noted that the tumor hypoxic fraction generally increased as the tumor size increased, in agreement with other published studies $(11,12)$. Early studies showing a relationship between tumor size and hypoxic fraction used a radiobiological assay of hypoxia (11). One of the most common radiobiological assays of hypoxia involves performing an in vivo/in vitro colony-forming assay on two experimental groups: mice breathing ambient air, and mice asphyxiated with nitrogen gas. The hypoxic fraction is calculated from the difference in cell survival between the air breathing group and the asphyxiated hypoxic group. Using assays such as these, it was generally found that in a variety of tumors weighing less than a gram, larger tumors exhibited more hypoxia than smaller tumors $(11,13-15)$. In the case of KHT sarcomas, it was noted that tumors larger than $0.7 \mathrm{~g}$ did not show a further increase in hypoxic fraction, indicating that some tumors may reach a plateau in their hypoxic fraction (14). In contrast with these studies, studies with both a rhabdosarcoma (16) and in a 9L line (17) were not able to show a dependence of hypoxic fraction on tumor size. Later studies used an invasive polargraphic needle electrode to assess the dependence of hypoxic fraction on tumor size. These studies found that in OCa-I, MCa-r, KHT, C3H mammary carcinoma and SCCVII tumors with weights ranging from 0.15 to $1.5 \mathrm{~g}$, the hypoxic fraction increased as the tumor grew (18-20). In the case of SCCVII (also used in this study), polarographic oxygen measurements showed that the hypoxic fraction (defined 
in that study as $\%$ volume with $\mathrm{pO}_{2}<5 \mathrm{mmHg}$ ) ranged from approximately 50 to $100 \%$ as the tumor grew from $150 \mathrm{~mm}^{3}$ to $1,500 \mathrm{~mm}^{3}$ (20). These measurements report hypoxia fractions greater than observed in the present study, where the hypoxic fraction (defined in this study as \% volume with $\mathrm{pO}_{2}<10 \mathrm{mmHg}$ ) was found to increase from 10 to $50 \%$ as the tumor grew from $500 \mathrm{~mm}^{3}$ to $3,500 \mathrm{~mm}^{3}$. In summary, most of the literature is consistent with the current finding that implanted animal tumors weighing less than a few grams exhibit an increased hypoxic fraction as the tumors grow.

Although most murine tumors exhibit a dependence of hypoxic fraction on tumor size, there are data suggesting that in the case of human tumors, the situation is more complex. Using F-MISO PET, hypoxia was measured in tumors of 12 patients with non-small cell lung cancer, where the tumor size ranged from $50 \mathrm{~cm}^{3}$ to $350 \mathrm{~cm}^{3}$ (21). In this study, no dependence of hypoxic fraction on tumor size was observed. In other studies in human uterine cervix cancers (22) and squamous cell carcinoma metastasis (23), hypoxia was measured using a polarographic needle. Again, no dependence of hypoxic fraction on tumor size was observed in either of these studies. Although these data are limited, they suggest that tumor size is not a valid single surrogate marker for hypoxia in the clinical setting. It is not completely clear why animal tumors exhibit a size-dependent hypoxia relationship and human tumors do not, but the difference may be related to the relative volume of murine versus human tumors. In general, the human tumors were at least $5 \mathrm{~g}$ (assuming water density) $(11,13,14)$, indicating that by the time human tumors are observed in the clinic, they may have surpassed a threshold for size-hypoxia dependence. Indeed, the hypoxic fraction cannot increase indefinitely, and murine KHT sarcomas exhibited a plateau in hypoxia above a weight of $0.7 \mathrm{~g}$ (14). Because tumor size, which is commonly measured during routine clinical scans, is apparently a poor marker for tumor hypoxia, novel biological imaging techniques must be developed in order to assess tumor hypoxia in humans.

During this study, it was observed that tumor hypoxia and the rate of Tempol reduction both increased as the tumor grew. The question remains if the increasing hypoxia observed during tumor growth actually caused Tempol to be reduced more quickly. In theory, it is possible that low oxygen levels in a tumor will result in a more reductive tumor microenvironment, and an important example involves the NADPH/NADP ${ }^{+}$redox pair (24). NADPH is an endogenous antioxidant, and its oxidation is catalyzed by an enzyme called NADPH oxidase (NOX) in a reaction that requires molecular oxygen. Furthermore, NADPH oxidase is overexpressed in some cancers (25), including SCC (26) (used in this study), implying that NOX activity may have affected NADPH levels in the tumor models used during this study. NADPH, in turn, is coupled to the reduction of GSSG to glutathione, which is the most abundant endogenous antioxidant. Thus, oxygen levels directly and indirectly may affect the intracellular levels of NADPH and GSH, both of which in turn affect the rate of Tempol reduction (7,27-30). Hypoxia may therefore cause the cellular milieu to become more reduced, which would result in a high rate constant for Tempol reduction.

Although increasing hypoxia may cause the intracellular environment to become more reduced through the mechanisms described above, those effects alone cannot account for the large increase in tumor redox observed in the current study. To put in perspective the findings of this study, it is useful to cite in vitro studies showing that compared to tumor cells exposed to $160 \mathrm{mmHg}$ oxygen, cells exposed to $0 \mathrm{mmHg}$ oxygen exhibit a $20-160 \%$ greater reduction rate constant $(31,32)$. Furthermore, in a RIF-1 tumor, it was found that the tumor $\mathrm{pO}_{2}$ varied between 2.5-15 $\mathrm{mmHg}$, but that the correlation between the nitroxide reduction rate constant and $\mathrm{pO}_{2}$ was weak $(\mathrm{r}=0.357)(33)$. It therefore seems unlikely that a small change in median $\mathrm{pO}_{2}$ of $5 \mathrm{mmHg}$ would result in a $100 \%$ increase in the reduction rate constant due only to the mechanisms described in the previous paragraph. These considerations suggest that the rapid Tempol reduction observed during this study may be due to factors more complex than simple accumulation of oxygen-dependent antioxidants due to low levels of molecular oxygen.

An alternative explanation of the apparent correlation between the hypoxic fraction and the rate of Tempol reduction observed in the current study could be a hypoxia-induced shift to glycolytic metabolism. Under conditions of hypoxia, signaling molecules such as hypoxia inducible factors (HIFs) become relatively abundant in the cellular milieu. The most commonly studied hypoxia inducible factor, HIF-1, is known to activate glycolytic enzymes and genes, thereby increasing glycolytic metabolism (34-36). Recently, glycolytic metabolism has been shown to provide resistance to $\mathrm{H}_{2} \mathrm{O}_{2}$ toxicity, decrease production of reactive oxygen species, and decrease the extent of oxidized DNA fragments. In other words, glycolytic metabolism has been shown to evoke an antioxidant response (34-36). Although the mechanism for this antioxidant response is not yet fully understood, it may also contribute to the more reductive tumor environment that was observed in this study.

In this study, it is observed that both hypoxia and the rate of nitroxide reduction increased as the tumor grew, suggesting that hypoxia and the rate of nitroxide reduction are correlated. Due to the prognostic value of hypoxia, this suggests that rapid nitroxide reduction may indicate poor response to cancer therapy. In conclusion, nitroxides such as Tempol may provide a clinically feasible magnetic resonance imaging based assay of hypoxia.

\section{Acknowledgements}

This study was supported by the Intramural Research Program of the Center for Cancer Research, National Cancer Institute, NIH.

\section{References}

1. Nordsmark M, Bentzen SM, Rudat V, Brizel D, Lartigau E, Stadler P, Becker A, Adam M, Molls M, Dunst J, Terris DJ and Overgaard J: Prognostic value of tumor oxygenation in 397 head and neck tumors after primary radiation therapy. An international multi-center study. Radiother Oncol 77: 18-24, 2005

2. Brizel DM, Dodge RK, Clough RW and Dewhirst MW: Oxygenation of head and neck cancer: changes during radiotherapy and impact on treatment outcome. Radiother Oncol 53: 113-117, 1999.

3. Hockel M, Knoop C, Schlenger K, Vorndran B, Baussmann E, Mitze M, Knapstein PG and Vaupel P: Intratumoral $\mathrm{pO}_{2}$ predicts survival in advanced cancer of the uterine cervix. Radiother Oncol 26: 45-50, 1993.

4. Roizin-Towle L and Hall EJ: Studies with bleomycin and misonidazole on aerated and hypoxic cells. Br J Cancer 37: 254-260, 1978.

5. Teicher BA, Lazo JS and Sartorelli AC: Classification of antineoplastic agents by their selective toxicities toward oxygenated and hypoxic tumor cells. Cancer Res 41: 73-81, 1981. 
6. Teicher BA,Holden SA, al-Achi A and Herman TS: Classification of antineoplastic treatments by their differential toxicity toward putative oxygenated and hypoxic tumor subpopulations in vivo in the FSaIIC murine fibrosarcoma. Cancer Res 50: 3339-3344, 1990.

7. Ilangovan G, Li H, Zweier JL, Krishna MC, Mitchell JB and Kuppusamy P: In vivo measurement of regional oxygenation and imaging of redox status in RIF-1 murine tumor: effect of carbogen-breathing. Magn Reson Med 48: 723-730, 2002.

8. Hyodo F, Soule BP, Matsumoto K, Matusmoto S, Cook JA, Hyodo E, Sowers AL, Krishna MC and Mitchell JB: Assessment of tissue redox status using metabolic responsive contrast agents and magnetic resonance imaging. J Pharm Pharmacol 60: 1049-1060, 2008.

9. Hyodo F, Matsumoto K, Matsumoto A, Mitchell JB and Krishna MC: Probing the intracellular redox status of tumors with magnetic resonance imaging and redox-sensitive contrast agents. Cancer Res 66: 9921-9928, 2006.

10. Matsumoto K, Subramanian S, Devasahayam N, Aravalluvan T, Murugesan R, Cook JA, Mitchell JB and Krishna MC: Electron paramagnetic resonance imaging of tumor hypoxia: enhanced spatial and temporal resolution for in vivo $\mathrm{pO}_{2}$ determination. Magn Reson Med 55: 1157-1163, 2006.

11. Shipley WU, Stanley JA and Steel GG: Tumor size dependency in the radiation response of the Lewis lung carcinoma. Cance Res 35: 2488-2493, 1975.

12. Stanley JA, Shipley WU and Steel GG: Influence of tumour size on hypoxic fraction and therapeutic sensitivity of Lewis lung tumour. Br J Cancer 36: 105-113, 1977.

13. Jirtle $R$ and Clifton KH: The effect of tumor size and host anemia on tumor cell survival after irradiation. Int J Radiat Oncol Biol Phys 4: 395-400, 1978.

14. Hill RP: An appraisal of in vivo assays of excised tumours. $\mathrm{Br}$ J Cancer (Suppl) 4: 230-239, 1980.

15. Siemann DW: Tumour size: a factor influencing the isoeffect analysis of tumour response to combined modalities. Br J Cancer (Suppl) 4: 294-298, 1980.

16. Reinhold HS and De Bree C: Tumour cure rate and cell survival of a transplantable rat rhabdomyosarcoma following $\mathrm{x}$-irradiation. Eur J Cancer 4: 367-374, 1968.

17. Wallen CA, Michaelson SM and Wheeler KT: Evidence for an unconventional radiosensitivity of rat 9L subcutaneous tumors. Radiat Res 84: 529-541, 1980.

18. De Jaeger K, Merlo FM, Kavanagh MC, Fyles AW, Hedley D and Hill RP: Heterogeneity of tumor oxygenation: relationship to tumor necrosis, tumor size, and metastasis. Int J Radiat Oncol Biol Phys 42: 717-721, 1998.

19. Khalil AA, Horsman MR and Overgaard J: The importance of determining necrotic fraction when studying the effect of tumour volume on tissue oxygenation. Acta Oncol 34: 297-300, 1995.

20. Milross CG, Tucker SL, Mason KA, Hunter NR, Peters LJ and Milas L: The effect of tumor size on necrosis and polarographically measured $\mathrm{pO}_{2}$. Acta Oncol 36: 183-189, 1997.

21. Rasey JS, Koh WJ, Evans ML, Peterson LM, Lewellen TK, Graham MM and Krohn KA: Quantifying regional hypoxia in human tumors with positron emission tomography of [18F]fluoromisonidazole: a pretherapy study of 37 patients. Int J Radiat Oncol Biol Phys 36: 417-428, 1996.

22. Weitmann HD, Gustorff B, Vaupel P, Knocke TH and Potter R: Oxygenation status of cervical carcinomas before and during spinal anesthesia for application of brachytherapy. Strahlenther Onkol 179: 633-640, 2003
23. Gatenby RA, Kessler HB, Rosenblum JS, Coia LR, Moldofsky PJ, Hartz WH and Broder GJ: Oxygen distribution in squamous cell carcinoma metastases and its relationship to outcome of radiation therapy. Int J Radiat Oncol Biol Phys 14: 831-838, 1988

24. Schafer FQ and Buettner GR: Redox environment of the cell as viewed through the redox state of the glutathione disulfide/ glutathione couple. Free Radic Biol Med 30: 1191-1212, 2001

25. Ilonen IK, Rasanen JV, Sihvo EI, Knuuttila A, Salmenkivi KM, Ahotupa MO, Kinnula VL and Salo JA: Oxidative stress in non-small cell lung cancer: role of nicotinamide adenine dinucleotide phosphate oxidase and glutathione. Acta Oncol 48: 1054-1061, 2009.

26. Czesnikiewicz-Guzik M, Lorkowska B, Zapala J, Czajka M, Szuta M, Loster B, Guzik TJ and Korbut R: NADPH oxidase and uncoupled nitric oxide synthase are major sources of reactive oxygen species in oral squamous cell carcinoma. Potential implications for immune regulation in high oxidative stress conditions. J Physiol Pharmacol 59: 139-152, 2008.

27. Iannone A, Tomasi A, Vannini V and Swartz HM: Metabolism of nitroxide spin labels in subcellular fractions of rat liver. II. Reduction by microsomes. Biochimica Biophysica Acta 1034: 285-289, 1990.

28. Finkelstein E, Rosen GM and Rauckman EJ: Superoxidedependent reduction of nitroxides by thiols. Biochim Biophys Acta 802: 90-98, 1984.

29. Yamada KI, Kuppusamy P, English S, Yoo J, Irie A, Subramanian S, Mitchell JB and Krishna MC: Feasibility and assessment of non-invasive in vivo redox status using electron paramagnetic resonance imaging. Acta Radiol 43: 433-440, 2002.

30. Kuppusamy P, Li H, Ilangovan G, Cardounel AJ, Zweier JL, Yamada K, Krishna MC and Mitchell JB: Noninvasive imaging of tumor redox status and its modification by tissue glutathione levels. Cancer Res 62: 307-312, 2002.

31. Davis RM, Matsumoto S, Bernardo M, Sowers A, Matsumoto K, Krishna MC and Mitchell JB: Magnetic resonance imaging of organic contrast agents in mice: capturing the whole-body redox landscape. Free Radic Biol Med 50: 459-468, 2011.

32. Samuni Y, Gamson J, Samuni A, Yamada K, Russo A, Krishna MC and Mitchell JB: Factors influencing nitroxide reduction and cytotoxicity in vitro. Antioxid Redox Signal 6: 587-595, 2004.

33. Takeshita K, Kawaguchi K, Fujii-Aikawa K, Ueno M, Okazaki S, Ono M, Krishna MC, Kuppusamy P, Ozawa T and Ikota N: Heterogeneity of regional redox status and relation of the redox status to oxygenation in a tumor model, evaluated using electron paramagnetic resonance imaging. Cancer Res 70: 4133-4140, 2010.

34. Kondoh H, Lleonart ME, Bernard D and Gil J: Protection from oxidative stress by enhanced glycolysis; a possible mechanism of cellular immortalization. Histol Histopathol 22: 85-90, 2007.

35. Kondoh H, Lleonart ME, Gil J, Wang J, Degan P, Peters G, Martinez D, Carnero A and Beach D: Glycolytic enzymes can modulate cellular life span. Cancer Res 65: 177-185, 2005.

36. Luo F, Liu X, Yan N, Li S, Cao G, Cheng Q, Xia Q and Wang H: Hypoxia-inducible transcription factor-1alpha promotes hypoxia-induced A549 apoptosis via a mechanism that involves the glycolysis pathway. BMC Cancer 6: 26, 2006. 